\title{
Do Gestalt Effects Show that We Perceive High-Level Aesthetic Properties?
}

\author{
Raamy Majeed \\ Draft: December 2017 \\ raamy.majeed@open.ac.uk
}

\begin{abstract}
Whether we perceive high-level properties is presently a source of controversy. A promising test case for whether we do is aesthetic perception. Aesthetic properties are distinct from low-level properties, like shape and colour. Moreover, some of them, e.g. being serene and being handsome, are properties we appear to perceive. Aesthetic perception also shares a similarity with gestalt effects, e.g. seeing-as, in that aesthetic properties, like gestalt phenomena, appear to 'emerge' from low-level properties. Gestalts effects, of course, are widely observed, which raises the question: do gestalt effects make it plausible that we (sometimes) perceive high-level aesthetic properties? Contra Stokes (forthcoming), this paper argues that they don't. This is interesting in its own right, but it also points to a more general lesson, namely we should resist the temptation to appeal to gestalt effects to argue for high-level perception.
\end{abstract}

KEYWORDS: High-Level Properties; Aesthetic properties; Rich Content; Liberal Content; Gestalt Psychology; Seeing-as

Whether we perceptually experience high-level properties is presently a source of controversy. A good, though neglected, test case for whether we do is aesthetic perception. Both common sense and dominant traditions in art criticism appear to treat aesthetic properties as perceptible. Moreover, these tend to pass as high-level properties on major ways of drawing the high-level vs. low-level distinction. For instance, they are properties which we can be aware of only via the neural processing of low-level properties (Lyons 2005). The ethereal quality of a Casorati painting, say, cannot be perceived without processing its shapes and colours.

It appears, then, that there are prima facie reasons for taking seriously the thesis we perceive high-level aesthetic properties. If this thesis actually turns out true, it would not only be a significant result, but a cause for surprise for many philosophers of mind sceptical of high-level perception. 
Stokes (forthcoming) argues it is true, and does so by appealing to the occurrence of gestalt effects, e.g. seeing-as. Gestalt effects are often employed in discussions of aesthetic experience, e.g. Sibley (1959), Walton (1970) and Arnheim (1974). They are also sometimes alluded to in discussions of high-level perception, e.g. Block (2014) and Helton (2016). However, aside from Stokes, there is a conspicuous absence of any discussion of the idea that lessons for high-level perception might be drawn from the analogy between perceiving aesthetic properties and gestalt effects. Stokes's paper is significant precisely because it offers the only articulation of this idea to date.

The present paper aims to explore this idea further, and does so by raising scepticism about Stokes’s proposal. In what follows, I outline Stokes’s argument (\$I), highlight an important distinction between types of gestalt effects ( $(I I)$, and draw on this distinction to explain why the argument doesn't work ( $(\mathrm{III})$. I conclude by drawing the opposite lesson to the one presently available: gestalt effects do not make it plausible that we perceive high-level aesthetic properties.

\section{The Argument from Seeing-As}

Stokes defends the thesis that we (sometimes) perceive high-level aesthetic properties by appealing to the following argument:

1. Seeing-as, e.g. seeing an image as a duck vs. seeing it as a rabbit, involves the representation of high-level properties.

2. Plausibly, the two overall experiences involved in seeing-as, e.g. seeing an image as a duck vs. seeing it as a rabbit, differ in phenomenology.

3. The most natural explanation for (2) is that seeing-as involves a difference in visual representation.

4. Therefore, seeing-as involves the visual representation of high-level properties.

5. Aesthetic properties, like being serene and being impressionist, are high-level properties.

6. Perceiving such aesthetic properties involves the same kind of perceptual gestalts as seeing-as.

7. Therefore, perceiving such aesthetic properties involves the visual representation of high-level properties. 
The argument is an inference to the best explanation, and attempts to make plausible the conclusion, “aesthetic reaction sometimes involves perceptual representation of high-level properties" (pg. 14-5). One may take issue with any number of its premises, but the point of interest for us is the part of the argument which exploits the similarity between gestalt effects and our aesthetic reactions. In other words, what concerns us is premise (6). As we shall see, this premise, as well as (1) and (4), are called into question on account of different ways of understanding gestalt phenomena.

\section{Types of Gestalt Effects}

Stokes's exposition of gestalt effects relies on the notion of seeing-as, which he takes to be exemplified by our experiences of the duck-rabbit (figure. 1) and the Rubin goblet (figure. 2). These examples are misleading. Nevertheless, explaining why they are so helps identify the precise way in which our aesthetic reactions are supposed to involve the same kind of perceptual gestalts as those involved in seeing-as.

The examples are misleading for the following reason. The phenomenon of seeing-as, exemplified by these examples, is a gestalt shift. All gestalt shifts are gestalt effects, but not all gestalt effects are gestalt shifts. The problem is gestalt shifts count as gestalt effects in two ways, which in turn obscures the precise feature they have in common with aesthetic reaction. (What such reaction has in common with gestalt effects, I shall argue, is a feature that is not particular to gestalt shifts).

Some background helps bring this out. The term 'gestalt' literally means shape or form, while Gestalt psychology is a theory premised on the primacy of perceiving the whole over its parts. As von der Heydt et al. observe, "structured wholes or Gestalten, rather than sensations, are the primary units of mental life. This was the key idea of the new and revolutionary Gestalt theory, developed by Wertheimer and his colleagues in Berlin" (2012: 1173). This idea is often spelt out by explaining how certain demonstrable visual effects conform to gestalt principles to do with perceptual grouping and figure-ground organisation.

Stokes's argument does not rely on Gestalt psychology being true, but simply the occurrence of certain gestalt effects. The theory, nonetheless, helps inform what counts as a gestalt effect. Such an effect is an effect that conforms to one or more gestalt principles; principles concerning figure-ground organisation and perceptual grouping. Gestalt shifts, as exemplified by our experiences of the duck- 
rabbit and the Rubin goblet, conform to two such principles. The obvious one is 'multistability'. This principle concerns how ambiguous figures, i.e. figures that can be seen as more than one image, cannot be viewed simultaneously, but rather shift back and forth between the various images.

This holds true for our experiences of the duck-rabbit and the Rubin goblet, as well as our experiences of simpler images, like the Necker cube (figure 3.). In the latter case, we see the image of a cube either as one where the bottom left square appears in the foreground while the top right one appears in the background, or vice versa. We cannot, however, see the image as one where both the bottom left and top right squares appear in the foreground; nor as one where both appear in the background. The image shifts back and forth between either square being in the foreground or the background. This is why effects such as these are described as gestalt shifts.

These examples also conform to another principle, viz: 'emergence'. According to this principle, we often see images primarily as a whole, as opposed to constructing them out of parts which we have to see first. For instance, in the duck-rabbit case, we see either an image of a duck or of a rabbit, but crucially, for whichever image we see, we appear to see it as a whole. That is, in contrast to first seeing the individual colours and shapes that compose the image, and then piecing together the image from such experiences. This principle goes to the very heart of gestalt psychology. But what's more, this principle is satisfied by visual effects that don't involve gestalt shifts. In other words, visual effects can conform to this principle without also conforming to the principle of multistability.

Consider the zebra image (figure. 4) by way of demonstration. When perceiving the image, we do not first see its parts, i.e. the various vertical and horizontal black lines, individually and then infer the whole, i.e. the zebra-shape, from these parts. Rather, the zebra-shape appears all at once. Our experience of the image, then, conforms to the principle of emergence. Nevertheless, it does so without conforming to the principle of multistability, as the figure is not ambiguous between two or more images.

This distinction between types of gestalt effects — based on the particular gestalt principles to which they conform - proves significant for how we are to understand (6). The premise states perceiving aesthetic properties, e.g. being serene and being impressionist, involves the same kind of perceptual gestalts as seeing-as. We now see that this could mean aesthetic perception conforms to the principe of 
emergence, the principle of multistability or both. An examination of aesthetic experience, nevertheless, reveals the relevant principle to be emergence.

In his exposition of (6), Stokes claims it is "natural" to describe a case where a child acquires the capacity to reliably attribute 'impressionist' to paintings 'in terms of a gestalt, even if that gestalt cannot be defined in rigorous terms" (pg. 6). Though a child's visual experiences of paintings before and after she learns how to discriminate impressionist paintings can (figuratively) be said to undergo a gestalt shift, her experience of any given painting doesn't have the feature of multistability. Looking at a Monet doesn't involve our experiences switching back and forth between two ambiguous figures, one that has the property being impressionist, the other which doesn't; or likewise, one that has the property being serene, the other which doesn't.

A charitable reading has it that the gestalt feature in common between seeing the duck-rabbit and seeing a Monet as impressionist is not multistability but something else. The following passage lends a clue:

What [sensory perception] can represent, and more or less accurately, is the way basic features of an object are organized. This is the phenomenal character that pops out when one shifts from seeing-as a duck to seeing-as a rabbit, and perhaps it is what pops out when one learns to recognize pine trees. (pg. 27) ${ }^{1}$

This suggests a child's visual experience of a painting when she acquires the capacity to reliably attribute 'impressionist' to paintings has the following characteristic: the way basic features of the painting are organised 'pop out' at her. The principle of gestalt theory most suitable to describe this effect is emergence. When she sees the painting, she does not piece together the organised whole from its basic features; rather, she perceives the whole all at once.

Plausibly, a gestalt effect, then, still occurs, even though it isn't literally a gestalt shift. Moreover, it does so precisely because there is a feature, viq. emergence, which this and other aesthetic reactions have in common with instances of seeing-as. This point is significant, as we now see the suitability of

\footnotetext{
${ }^{1}$ The mention of recognising pine trees is a reference to Siegel (2006), who argues that a difference in visual experience is brought about when one learns how to discriminate pine trees.
} 
comparing aesthetic reactions with gestalt effects (by way of arguing for high-level aesthetic perception) rests on both exhibiting the feature of emergence.

\section{Why the Argument Doesn't Work}

Stokes's argument appeals to a similarity between gestalt effects and aesthetic reaction to argue we can perceive high-level aesthetic properties. We now see the relevant similarity to be emergence. This focus on emergence helps us identify two distinct ways of disambiguating the phenomenon of seeing-as, both of which ultimately render the argument implausible. The difference has to do with what is supposed to emerge as the relevant gestalten. On a modest reading, the gestalten which emerge or 'pop out' are certain organised shape properties. For example, in the duck-rabbit case, what emerge are the shape-properties being organised like a duck and being organised like a rabbit. By contrast, on an immodest reading, what emerge are something above and beyond organised shape properties. They might be artificial kind properties, e.g. being a duck image and being a rabbit image, or being a duck representation and being a rabbit representation.

To be clear, the above distinction rests not on the shifting nature of the gestalten, but the kinds of gestalten at issue. On the modest interpretation, the gestalten are organised shapes, whereas in the immodest case, they are something else. How to articulate what emerges in this case is hard to say. This, in part, is due to the difference between the two interpretations being typically obscured in discussions of seeing-as, especially those which employ the duck-rabbit image as an example. The difference can be brought out by comparing this to the Necker cube. In the latter case, what emerges are just the organised shape properties. We see the image as one of a cube, in particular a cube that oscillates between one where either the left or right square is in the foreground. Crucially, the gestalten that appear are only organised shape properties. By comparison, in the duck-rabbit case, while the two gestalten that emerge in the first instance are organised shape properties, i.e. being organised like a duck and being organised like a rabbit, it also arguably involves two further gestalten, e.g. being a duck representation and being a rabbit representation. 
A way to illustrate this is to claim seeing organised shape properties doesn't require conceptpossession, whereas seeing properties of the latter kind does. ${ }^{2}$ One can, for instance, see the shape, which happens to be the shape of a goblet, without recognising that this shape is the shape of a goblet. One can't, however, see that this shape is the shape of a goblet without possessing the concept of a goblet. Of course, any claims about concept-possession are bound to be contentious. But this example is merely illustrative, and is by no means required to make the distinction between the two types of gestalten which might be at play in some cases of seeing-as, e.g. the duck-rabbit image. In what follows, I treat this as a distinction between seeing organised shape properties and seeing certain artificial kind properties — though I assume the ensuing points can also be made on other ways of capturing this distinction.

The advantage of the modest reading of seeing-as, where the gestalten are (only) organised shape properties, is that such effects are widely observed. Whether these effects conform exactly to gestalt principles, as they have been described by various gestalt theorists, is open to dispute. However, that these effects occur, I take it, isn't. This means insofar as the perception of aesthetic properties involves the same kind of perceptual gestalts as seeing-as, we have good grounds to suppose that we do, genuinely, perceive aesthetic properties.

The problem with this reading is twofold. First, it threatens the role (6) is supposed to play in the argument. Simply put, it is contentious whether organised shape properties can be described as high-level properties. This makes (1) — that seeing-as involves the representation of high-level properties - contentious. But it also means even if (6) were true, it won't, subsequently, help establish that we can perceive high-level aesthetic properties — at least not in an uncontroversial manner.

To elaborate, organised shape properties are dependent on low-level (non-organised) shape properties, and thereby count as high-level properties in one sense. But shape properties are paradigm examples of low-level properties, and so even organised shape properties count as low-level properties in another sense. ${ }^{3}$ For the purposes of this paper, I wish to bypass the thorny issue of whether organ-

\footnotetext{
2 This is orthogonal to the thesis that all perception involves some sort of conceptual content.

${ }^{3}$ Following Brogaard and Chomanaski (2015), my preference is to treat the high-level vs. low-level distinction as a relative notion, where the organised shape properties are at a higher-level than the shape properties on which they depend.
} 
ised shape properties count as high-level properties. This is because adjudicating the matter will prove difficult given that how we ought to understand the high-level vs. low-level distinction will depend on your theoretical concerns. What is worth noting is one of the concerns here is to understand the distinction in a way that respects the present controversy over whether we perceive high-level properties. I doubt those who deny rich perceptual content wish to also deny the occurrence of gestalt effects concerning perceptual grouping and figure-ground organisation. So the present controversy doesn't seem to be one about whether we can perceive high-level properties, where paradigm examples of such properties include organised shape properties. The overall consequence of the modest reading, then, is that (6) being true would, at best, only lend contentious support for the thesis that we can perceive highlevel aesthetic properties.

The second problem is more damning, as here we find the modest reading actually also renders (6) implausible. How it precisely does so will depend on the aesthetic properties at issue. Stokes provides two examples of aesthetic properties, being serene and being impressionist. These properties aren't on a par. The dominant tradition in aesthetics is to treat aesthetic properties as those properties picked out by aesthetic terms, where these terms, following Sibley (1959), are viewed as terms which require perceptiveness in order for their application. Sibley's own list includes lifeless, serene, somber, dynamic, delicate, moving, trite, sentimental, tragic, handsome, and garish. Style properties, e.g. being impressionist and being fauvist, aren't paradigm aesthetic properties. They have to do with how we categorise artworks, as opposed to how we might describe them. Contra Stokes, this makes them artificial kind properties. But note, they are a special class of such properties, for their detection seems to require perceptiveness. For this reason, we may, following Stokes, also classify them as aesthetic properties. The labels don't really matter. What does is there being a difference between style properties and paradigmatic aesthetic properties; a difference which proves significant when assessing the plausibility of (6).

One of the aesthetic properties mentioned, i.e. being impressionist, is an artificial kind property. This is the kind of property ruled out from being gestalten on the modest reading of seeing-as. So on this reading, we can't claim seeing aesthetic properties, qua properties such as being impressionist, involves the same kind of perceptual gestalts as seeing-as. Premise (6), it turns out, is implausible from the getgo. 
A different challenge to (6) presents itself when we focus on paradigm examples of aesthetic properties, e.g. being serene. Like style properties, paradigm aesthetic properties, insofar as they are highlevel properties, are properties that depend on low-level properties, e.g. shape and colour. The exact nature of this dependence is a matter of dispute. Nevertheless, the fact that we posit high-level properties, for ontological or theoretical reasons, is demonstrative that they aren't properties identical with the low-level properties on which they depend. Or, if they are thus identical, these identities aren't obvious. They require the mastery of concepts, or something similar. Sibley for instance, in his discussion of aesthetic concepts, argues aesthetic judgements require the exercise of taste. Regardless of how we fill in the details, the point is, insofar as aesthetic properties are high-level properties, any inference from perceiving low-level properties, e.g. shape and colour, even organised shape properties, to perceiving high-level aesthetic properties, e.g. being serene, isn't justified; at least, not without further qualification.

What is required is some further set of conditions that would license such inferences. Such conditions aren't hard to come by. They might involve acquiring the relevant concepts. Or gaining familiarity with artworks of a certain type such that we are able to recognise certain similar patterns in them even though they might differ dramatically in shape and colour, e.g. consider the differences between the works of Monet and Pissarro despite both being impressionist and often serene. But how we fill in the details here will prove crucial to the plausibility of whether we actually see high-level aesthetic properties. The condition of concept possession, for instance, makes it easy to deny high-level perception because the sceptic can argue even though we make aesthetic judgements concerning high-level properties, such judgements are really post-perceptual judgements we make on the basis of (only) seeing low-level properties. Likewise, if the condition is one of, say, familiarity, the sceptic could argue the patterns one sees are only patterns concerning low-level properties, e.g. certain similarities in organised patterns of shape and colour. The whole point of comparing aesthetic reaction to visual gestalts was that we are supposed to pre-empt such sceptical responses on the basis of the plausibility of the latter. But if the relevant inferences from gestalt effects to aesthetic reaction can only be made by stipulating further conditions, conditions which make room for well-known sceptical responses, we lose the incentive for making the comparison as means to argue for high-level aesthetic perception. 
The immodest reading of seeing-as bypasses these worries because the gestalten in some cases are found to be high-level properties, e.g. artificial kind properties like being a duck representation. The advantage of this reading is since the perception of high-level properties is a feature of some visual gestalts, inferences from such visual gestalts to perceiving high-level aesthetic properties is licensed on the basis that both experiences are of the same kind. For instance, if we can actually see the property being a goblet representation from organised patterns of shape and colour in the Rubin goblet, insofar as aesthetic reaction is literally a form of perceptual gestalt, we can also, plausibly, perceive properties like being serene and being impressionist.

The problem with this is whether visual gestalts actually involve the perception of high-level properties is very much open to dispute. In other words, (4) is contentious. Visual gestalts are demonstrable. However, what isn't is whether these effects involve merely the perception of organised patterns of shape and colour, or something above and beyond this. The duck-rabbit gestalt effect, for example, is widely reported, but it remains unclear whether this effect is to be interpreted as one where we actually see the property being a duck representation over and above the organised shape property being organised like a duck. All the reasons for being sceptical of high-level perception will motivate scepticism about such an interpretation. Subsequently, this reading of seeing-as is as controversial as the central controversy over whether we can see high-level properties.

Though this paper concerns the use of gestalt effects to argue for the perception of high-level aesthetic properties, the above point also speaks to possible attempts to employ gestalt effects to argue for high-level perception more broadly. The central worry is any interpretation of gestalt effects, which would lend support for the thesis that we perceive high-level properties, would be as controversial as the thesis itself. To reiterate, this is because arguments that tell against the thesis also tell against such an interpretation. Hence, though gestalt effects are common, it is doubtful that high-level theorists can further their cause by appealing to them.

In summary, premise (6) states that perceiving some aesthetic properties involves the same kind of perceptual gestalts as seeing-as. This is a premise in Stokes's argument for the thesis that we sometimes perceive high-level aesthetic properties. In this paper, we saw there are two readings of seeing-as. First, a modest reading which is plausible in and of itself, but which makes (6) implausible, and thereby 
doesn't lend any additional credibility to the aforementioned thesis. Second, a less modest reading, one that makes (6) plausible, but which is controversial itself, and thus ultimately won't make the thesis any more plausible either. The upshot is we reach a negative verdict: gestalt effects do not make it plausible that we perceive high-level aesthetic properties. This is interesting in its own right, but this paper also hints at a more general take-home message, namely we should resist the temptation to appeal to gestalt effects to argue for high-level perception.

\section{REFERENCES}

- Arnheim, R. (1974). Art and Visual Perception: A Psychology of the Critical Eye - The New Version. Berkeley, Los Angeles, London: University of California Press.

- Block, N. (2014). 'Seeing-As in the Light of Vision Science'. Philosophy and Phenomenological Research 89 (1):560-572.

- Brogaard, B.\& Chomanski, B. (2015). 'Cognitive Penetrability and High-Level Properties in Perception: Unrelated Phenomena?' Pacific Philosophical Quarterly 96 (4):469-486.

- Helton, G. (2016). 'Recent Issues in High-Level Perception’. Philosopby Compass 11 (12):851-862.

- Lyons, J. (2005). 'Clades, Capgras, and Perceptual Kinds'. Philosophical Topics 33(1): 185-206.

Sibley, F. (1959). 'Aesthetic concepts'. Philosophical Review 68 (4):421-450.

- Siegel, S. (2006). 'Which Properties Are Represented in Perception?' In T. S. Gendler and J. Hawthorne (eds.), Perceptual Experience. Oxford: Oxford University Press.

- Stokes, D. (forthcoming). 'Rich Perceptual Content and Aesthetic Properties'. In A. Bergqvist \& R. Cowan (eds.), Evaluative Perception. Oxford: Oxford University Press. Online version: https://philpapers.org/archive/STORPC.pdf

- von der Heydt, R. et. al. (2012). 'A Century of Gestalt Psychology in Visual Perception: I. Perceptual Grouping and Figure-Ground Organization'. Psychological Bulletin: American Psychological Association Vol. 138 (6): 1172-1217.

- Walton, K. (1970). 'Categories of Art'. Philosophical Review 79: 334-67. 


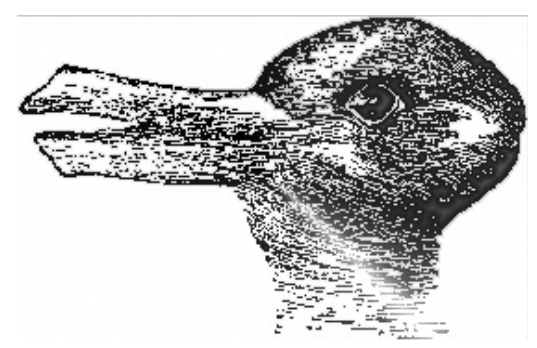

Figure 1.

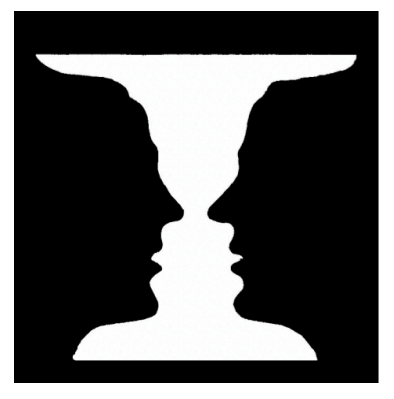

Figure 2.

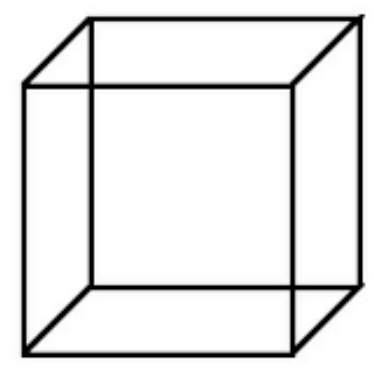

Figure 3.

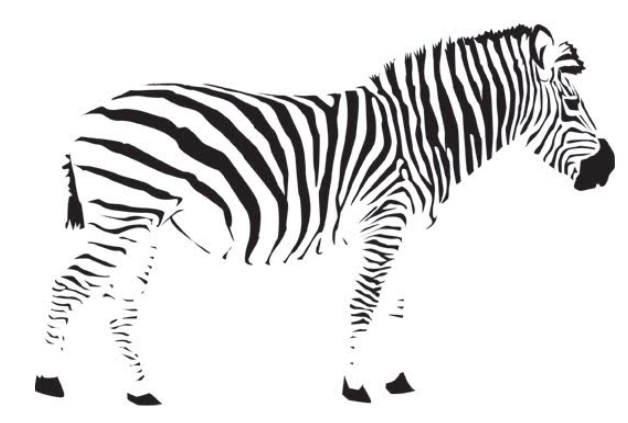

Figure 4. 